\title{
La nutrición materna y la programación metabólica: el origen fetal de las enfermedades crónicas degenerativas en los adultos
}

\section{Maternal nutrition and metabolic programming, the fetal origins of chronic degenerative diseases in adults}

\author{
Asdrúbal Aguilera Méndez \\ Universidad Michoacana de San Nicolás de Hidalgo, México \\ amendez@umich.mx \\ (D) http://orcid.org/0000-0003-0326-2068
}

Recepción: 4 de junio de 2019

Aprobación: 26 de septiembre de 2019

(c) (i) $\odot$

\begin{abstract}
Resumen
Diversas investigaciones han demostrado que alteraciones nutricionales en las madres influyen para que los hijos padezcan enfermedades crónicas no transmisibles en la edad adulta. Estas enfermedades son patologías de lenta evolución y no contagiosas como las involucradas en el síndrome metabólico que incrementa el riesgo de padecer diabetes tipo 2 y enfermedades cardiovasculares. En esta revisión se analizan los conceptos de síndrome metabólico, programación metabólica fetal, epigenética y factores nutricionales (desnutrición y sobrealimentación) relacionados con alteraciones en el desarrollo temprano de los individuos que condicionan en la edad adulta la aparición de enfermedades crónicas no transmisibles. Por desgracia, las perspectivas sobre el panorama de una reducción en estas enfermedades tanto en México como en el resto del mundo son poco alentadoras.
\end{abstract}

Palabras ClaVe: Nutrición materna, programación metabólica, enfermedades crónicas no transmisibles, síndrome metabólico, epigenética.

\begin{abstract}
Several studies have demonstrated that nutritional alterations in mothers has influence on the risk of non-communicable diseases in adulthood of progeny. These non-contagious pathologies of slow evolution, such as those involved in the metabolic syndrome, increase the risk of suffering from type 2 diabetes and cardiovascular diseases. In this review, we will discuss the concepts of metabolic syndrome, fetal metabolic programming, epigenetics, nutritional factors (malnutrition and overfeeding), related to alterations in the early development of individuals, that condition the emergence of non-communicable diseases in adulthood. Unfortunately, the perspectives on the panorama of a reduction in these diseases both in Mexico and at a global level is not very encouraging.
\end{abstract}

KEYWORDS: Maternal nutrition, metabolic programming, chronic non-communicable diseases, metabolic syndrome, epigenetics.

\section{INTRODUCCIÓN}

Diversos estudios epidemiológicos y experimentales apoyan fuertemente que alteraciones durante etapas críticas en el desarrollo de los individuos, como el crecimiento intrauterino, la lactancia y los primeros años de vida, son condicionantes para el riesgo de padecer enfermedades crónicas no transmisibles en la edad adulta (Halfon et al., 2018). Estas enfermedades son patologías de lenta evolución y no contagiosas, como la resistencia a la insulina, la dislipidemia, la hipertensión, la esteatosis hepática o hígado graso y la obesidad, que a su vez están involucradas en el síndrome metabólico, el cual incrementa el riesgo de padecer diabetes tipo 2, así como enfermedades cardiovasculares; actualmente estos padecimientos forman parte de las principales causas de muerte en México y en el mundo (Barba-Evia, 2018; WHO, 2015; GBD, 2017). Una forma de explicar la relación entre las alteraciones en etapas tempranas del desarrollo y las enfermedades crónicas no transmisibles en la edad adulta es la participación de mecanismos moleculares que modifican químicamente el ADN sin cambios en su secuencia; a esto se le conoce como epigenética, la cual origina una programación metabólica 
y plantea la hipótesis sobre los Origenes del desarrollo de la salud y las enfermedades en el adulto (Fleming et al., 2018). Esta programación condiciona la aparición de enfermedades crónicas no transmisibles en la edad adulta de los individuos cuando las condicionas ambientales relacionadas con la nutrición y el ejercicio no son las adecuadas y además se pueden heredar a la descendencia (Wang et al., 2018).

\section{LAS ENFERMEDADES CRÓNICAS NO TRANSMISIBLES}

En el último siglo se ha observado un aumento en todo el mundo de las enfermedades crónicas no transmisibles. Estas enfermedades son padecimientos con un desarrollo muy lento, no son contagiosas y en la mayoría de los casos son incurables, pero sí tratables. El síndrome metabólico representa el mejor ejemplo de este tipo de enfermedades, además de que es un importante problema de salud pública tanto en México (Barba-Evia, 2018) como en el mundo (WHO, 2015; GBD, 2017). Este síndrome es definido como una constelación de factores bioquímicos, clínicos y metabólicos interconectados que incrementan el riesgo de padecer enfermedades cardiovasculares (hipertensión, enfermedad cardiaca coronaria, cerebrovascular, arterial periférica, cardiopatía reumática, cardiopatía congénita e insuficiencia cardiaca) y diabetes tipo 2 (McCracken et al., 2018) (figura 1).

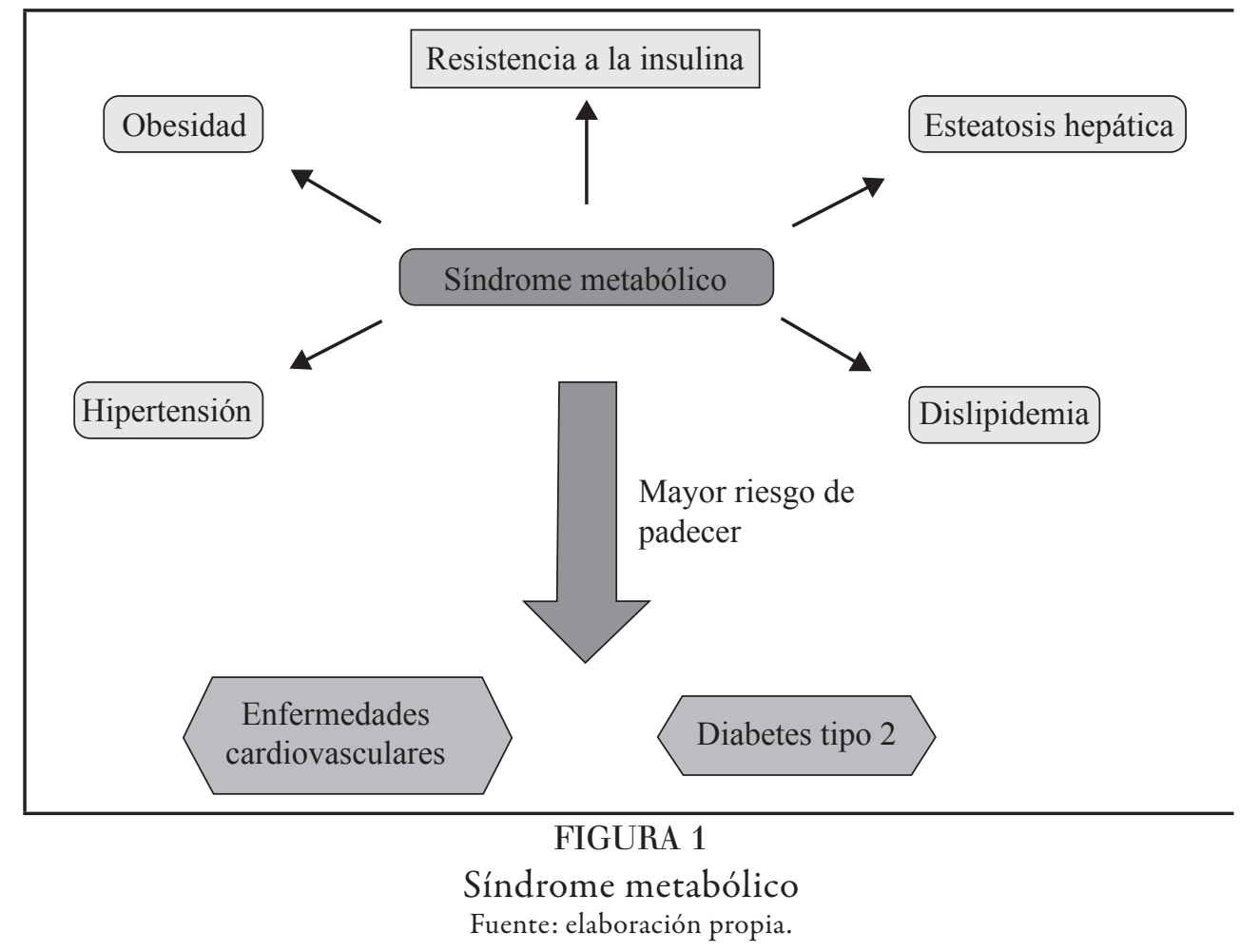

Se manifiesta clínicamente por la presencia de alteraciones en la tolerancia a la glucosa, resistencia a la insulina, hipertensión arterial, esteatosis hepática (hígado graso), obesidad, dislipidemia y microalbuminuria. Además de los factores genéticos que predisponen, los malos hábitos alimenticios y un estilo de vida sedentario también contribuyen a la pandemia del síndrome metabólico. Sin embargo, la gran dimensión del problema indica que existen otros tantos que deben tener una importante función en su origen (McCracken et al., 2018). Numerosos estudios epidemiológicos en personas y experimentales en animales sugieren que los desequilibrios nutricionales durante las primeras etapas del desarrollo de los individuos (gestación, lactancia y primeros años) tienen efectos posteriores sobre la salud y pueden ser responsables de la programación metabólica fetal y además estar asociadas a enfermedades del síndrome metabólico en la vida adulta (Wang et al., 2018). 


\section{Programación metabólica fetal y los orígenes del desarrollo de la salud y las ENFERMEDADES EN EL ADULTO}

El crecimiento y desarrollo intrauterino está dado por una serie de mecanismos activos y complejos regulados por las instrucciones genéticas adquiridas durante la concepción (Patel y Srinivasan, 2011). En este sentido, el ambiente intrauterino es una de las etapas de mayor plasticidad porque se forman y maduran los órganos y tejidos. La relación entre una buena calidad del medio ambiente intrauterino y el futuro riesgo de enfermedades en el adulto fueron descritas por primera vez por Forsdahl en 1977 (Forsdahl, 1977). Posteriormente, Barker y colaboradores reportaron que el peso bajo al nacer se asociaba con un mayor riesgo de muerte por enfermedades cardiovasculares (Barker y Osmond, 1986; Baker, 1995). Por lo tanto, estos estudios dieron la pauta para la noción de que la fisiología materna y paterna, su composición corporal, la calidad nutricional y el ejercicio durante el periodo de preconcepción y durante el embarazo tienen efectos profundos y permanentes en el riesgo de padecer enfermedades en la edad adulta de la descendencia. Además, esta noción ha recibido un gran número de evidencias experimentales de modelos animales, así como de estudios epidemiológicos en humanos (Wang et al., 2018).

De esta manera, surge el concepto de la programación metabólica fetal, la cual se entiende como un periodo en la etapa intrauterina donde determinados desequilibrios establecen adaptaciones metabólicas que permiten la sobrevivencia del individuo, pero dichas modificaciones condicionan una susceptibilidad para la aparición de enfermedades asociadas al síndrome metabólico en la edad adulta. Este concepto ha permitido generar la hipótesis sobre los "Orígenes del desarrollo de la salud y las enfermedades en el adulto" (DOHaD-Developmental Origins of Health and adult Disease), la cual se basa en el hecho de que las etapas (maduración del gameto, fertilización y desarrollo embrionario temprano) del periodo periconcepcional son vulnerables a diversos factores que producen cambios mediante respuestas compensatorias adaptativas y que pueden manifestarse en la vida adulta del individuo como enfermedades crónicas no transmisibles relacionadas con el síndrome metabólico (Velazquez et al., 2019; Fleming et al., 2018).

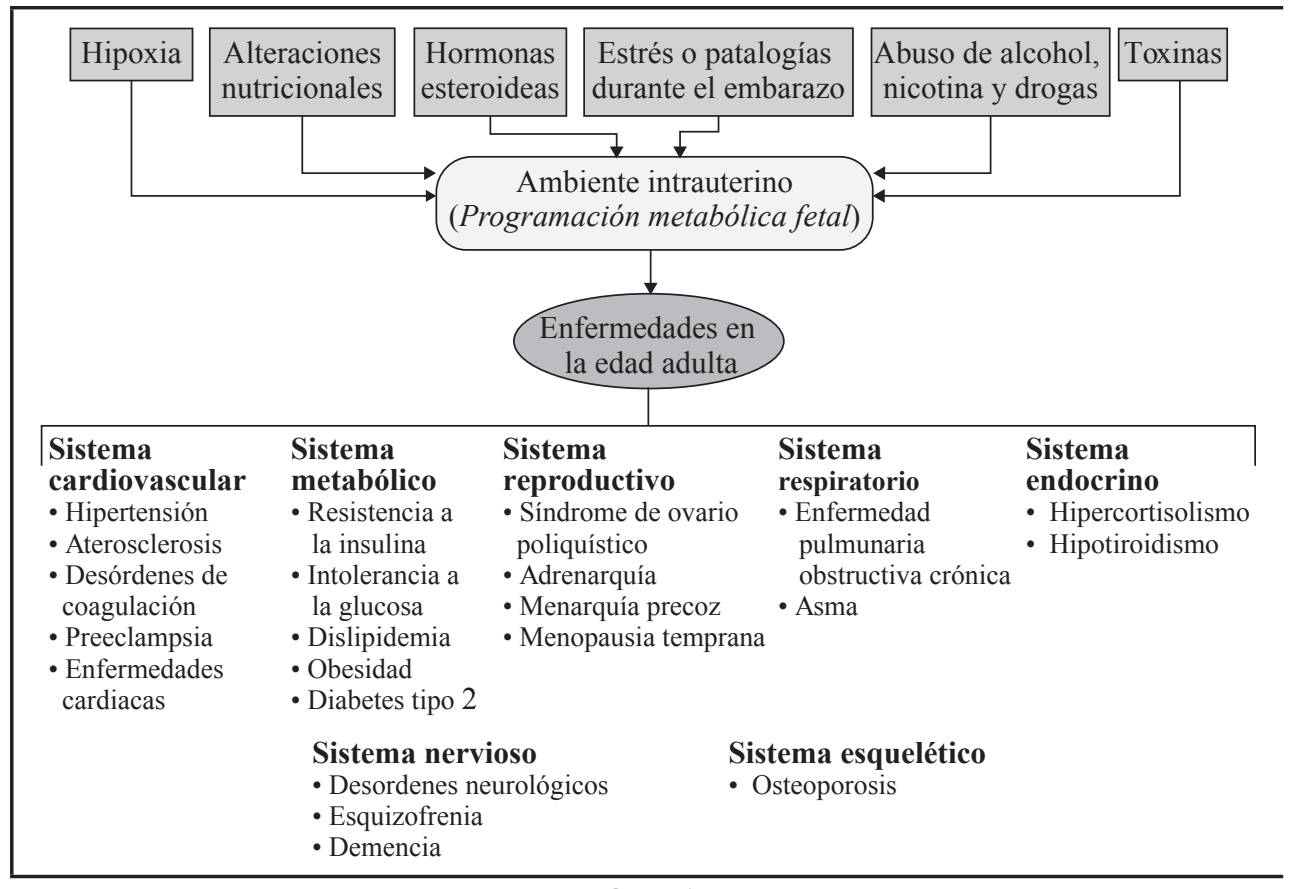

FIGURA 2

Factores que inducen la programación metabólica fetal y su relación con enfermedades en la edad adulta Fuente: elaboración propia. 
Los factores que inducen a la programación metabólica fetal y que condicionan la aparición de enfermedades asociadas al síndrome metabólico en la edad adulta son variados, y aún no son totalmente conocidos. Entre los más importantes se encuentran las alteraciones en la nutrición materna (desnutrición o sobrealimentación), pero también existen otros factores como la exposición a hormonas esteroideas, el estrés o patologías durante el embarazo (diabetes gestacional, preeclampsia, enfermedades virales o bacterianas), toxinas, hipoxia, abuso de alcohol, nicotina y drogas (Fowden et al., 2006).

Los factores mencionados, y los cuales inducen una programación metabólica, han demostrado tener efectos deletéreos a largo plazo en diferentes sistemas fisiológicos en la edad adulta como a) el sistema cardiovascular (relacionado con enfermedades como la hipertensión, la aterosclerosis, desórdenes de la coagulación, preeclampsia y enfermedades cardiacas, $b$ ) el sistema metabólico (relacionado con la resistencia a la insulina, la intolerancia a la glucosa, la dislipidemia, la obesidad y diabetes tipo 2), c) el sistema reproductivo (relacionado con el síndrome de ovario poliquístico, la adrenarquía, la menarquía precoz y la menopausia temprana, $d$ ) el sistema respiratorio (relacionado con la enfermedad pulmonaria obstructiva crónica y asma), $e$ ) el sistema endocrino (relacionado con el hipercortisolismo e hipotiroidismo), f) el sistema nervioso (relacionado con desordenes neurológicos, esquizofrenia y demencia) y $g$ ) el sistema esquelético (relacionado con la osteoporosis) (figura 2) (Fowden et al., 2006; Carpinello et al., 2018). Es importante recalcar que la mayoría de las enfermedades mencionadas están relacionadas con el síndrome metabólico.

Otro factor a considerar es el de los genes ahorradores, donde la expresión de éstos hace más eficiente el almacenamiento de energía en condiciones de poca abundancia nutricional y se ha observado en ciertos grupos étnicos. Por ejemplo, los indios pimas de Sonora viven en un ambiente de restricción nutricional relacionada con la pobreza y las labores del campo; son visiblemente delgados. En cambio, los indios pimas que habitan en Estados Unidos, aunque tienen el mismo origen genético, habitan en lugares donde se presenta exceso nutricional, trabajan en casinos y hacen poco ejercicio, por lo que presentan problemas de obesidad y diabetes. Con este ejemplo, se ha demostrado cómo este genotipo relacionado con genes ahorradores en un entorno abundante puede resultar nocivo, pues propicia la aparición de enfermedades crónicas no transmisibles relacionadas con el síndrome metabólico (Velazquez et al., 2019; Fleming et al., 2018). Por lo tanto, la programación metabólica relaciona la fisiopatología del síndrome metabólico con la compleja interacción de genes de alta susceptibilidad (genes ahorradores) que, junto con el ambiente intrauterino, predisponen la condición patológica antes que dicha enfermedad se manifieste (Wang et al., 2018).

\section{La EPIGENÉTICA EN LA PROGRAMACión METABóLICA}

Una creciente evidencia demuestra que los mecanismos epigenéticos son responsables de la programación metabólica durante el desarrollo y crecimiento en las primeras etapas de la vida de los individuos. Al respecto, la epigenética se define como los cambios en las expresión de los genes que suceden sin modificaciones en la secuencia del ADN y que generan un fenotipo específico (Burton y Lillycrop, 2019). Los principales mecanismos epigenéticos que regulan la expresión de genes en mamíferos son a) la metilación del ADN en la base púrica citocina, $b$ ) modificaciones covalentes (acetilación y metilación) en los aminoácidos de las histonas, y c) los RNAs no codificantes, como los microRNAs (miRNAs) (Milagro y Martínez, 2013; Zalts y Shomron, 2011).

Las ventanas epigenéticas se presentan en algunas etapas de la vida, como en el desarrollo embrionario, la lactancia y los primeros años de vida ( $1-5$ años principalmente), en los cuales existen una mayor susceptibilidad a modificaciones epigenéticas (modificaciones en el ADN y las histonas). La plasticidad del epigenoma permitiría una mejor adaptación a las condiciones del medio y prepararía el metabolismo del feto para las futuras condiciones nutricionales que le esperan (Burton y Lillycrop, 2019). Por lo tanto, la epigenética ayuda a comprender la relación entre la programación metabólica que establece las adaptaciones metabólicas en el organismo para condiciones ambientales específicas adversas y el desarrollo de enfermedades crónicas en el adulto, procesos que interactúan 
además con la nutrición materna (Taylor-Baer y Herman, 2018) y otros factores ambientales mencionados. Este contexto da bases sólidas para la hipótesis sobre los orígenes del desarrollo de la salud y las enfermedades en el adulto.

Se han realizado estudios que demuestran modificaciones epigenéticas en los espermatozoides de individuos que han sido expuestos a cambios desfavorables en su estilo de vida, lo cual trae consigo sobrepeso u obesidad (Velazquez et al., 2019), y que podrían condicionar a los hijos de estos individuos a ser susceptibles a padecer enfermedades relacionadas con el síndrome metabólico en su vida adulta si las condiciones de vida no son las adecuadas. Además, los procesos epigenéticos tienen la cualidad de memoria celular o heredabilidad transgeneracional y están implicados en la diferenciación celular espacial y temporal de las células totipotentes (Hsu y Tain, 2019). Existen varios estudios en animales que demuestran cómo las consecuencias adversas en el ambiente intrauterino tienen una herencia transgeneracional y están relacionadas con mecanismos epigenéticos donde existe una expresión alterada de los genes (Skinner, 2008).

\section{Factores nutricionales en la programación metabólica fetal}

La nutrición es indispensable en el crecimiento y desarrollo de los individuos y se manifiesta como un aumento (balance positivo), mantenimiento (balance neutro) o disminución (balance negativo) de la masa y del volumen corporal. Además, la nutrición se debe adecuar a las necesidades del organismo en relación con el cambio de forma, función y composición (Márquez-González et al., 2012). El crecimiento y el desarrollo del feto están determinados por tres factores: a) el estado nutricional de la embarazada, $b$ ) la función placentaria y c) la capacidad del feto para utilizar los nutrientes. Las alteraciones en la alimentación materna (desnutrición o sobrealimentación) durante las etapas tempranas del desarrollo pueden producir alteraciones en el crecimiento fetal, disminución o aumento dependiendo del insulto (sobrealimentación o desnutrición). También alteraciones en la placenta causan una disminución en el crecimiento fetal; sin embargo, actualmente no está claro el papel de la placenta en la programación metabólica fetal (Barrera-Reyesa y Fernández-Carrocera, 2015).

Se ha demostrado que las alteraciones nutricionales durante el embarazo pueden desencadenar respuestas adaptativas que pueden llevar a la pérdida de células (nefronas, cardiomiocitos, células pancreáticas, células de músculo esquelético, etc.) en diferentes órganos a expensas de mantener el desarrollo de otros órganos, como el cerebro. Estos cambios adaptativos son necesarios para la supervivencia en un ambiente nutricional adverso en su vida perinatal, pero condicionan o programan en los individuos una menor capacidad metabólica funcional para su vida adulta cuando son expuestos a un ambiente nutricional desfavorable (alimentación rica en carbohidratos y grasas saturadas) y a la falta de ejercicio (Marciniak et al., 2017; Carpinello et al., 2018).

Durante la nutrición materna existen dos condiciones nutricionales que determinan la programación metabólica fetal y el origen del desarrollo de la salud y las enfermedades en el adulto (Maliqueo y Echiburú, 2014).

a) La desnutrición: es una condición patológica inespecífica, sistémica y reversible en potencia debido a la deficiente utilización de los nutrimentos por el organismo y presenta diferentes grados de intensidad (MárquezGonzález et al., 2012). Los primeros estudios epidemiológicos en relacionar una deficiente nutrición materna y sus efectos sobre la salud en la vida adulta del hijo se realizaron en sujetos que sufrieron la hambruna holandesa de finales de la Segunda Guerra Mundial (1944 a 1945) (Roseboom, 2019). Los hombres que padecieron la hambruna en la etapa postnatal temprana presentaron una menor tasa de obesidad en la vida adulta en comparación con aquellos expuestos durante la primera mitad del embarazo. De forma similar, los individuos expuestos a la gran hambruna en China (1959 a 1961), en específico durante el primer trimestre, incrementaron el riesgo de padecer hipertensión arterial (Fleming et al., 2018). Estas observaciones han sido corroboradas en modelos animales (ovejas, ratas y ratones), en los cuales se ha demostrado que la restricción calórica y la deficiencia de micronutrientes (vitaminas y minerales) durante la gestación induce el desarrollo de obesidad, diabetes e hipertensión arterial en las crías en la edad adulta (Velazquez et al., 2019; Fleming et al., 2018). Además, se han observado cambios en la expresión de genes que codifican para hormonas y sus receptores (Perrone et al., 2016). 
Resulta interesante que los animales sometidos a una restricción calórica en etapas tardías de la gestación presentan alteraciones cardiovasculares, pero no desarrollan hipertensión, lo que pone de manifiesto el concepto de etapas críticas específicas en el establecimiento de la programación metabólica fetal (Maliqueo y Echiburú, 2014). Aunque son varios órganos y tejidos que participan en el control de la presión arterial, el riñón es particularmente susceptible a la desnutrición intrauterina. Varios estudios han demostrado una reducción en el número de nefronas, aumento del estrés oxidativo, activación del sistema renina-angiotensina y cambios en el transporte de sodio, lo cual propicia la aparición de alteraciones en la presión arterial (Tain et al., 2015).

b) La sobrealimentación: cuando existe un excedente en el consumo de calorías en relación con el gasto energético se presentan patologías por malnutrición por exceso. Se ha observado en estudios experimentales en animales que al exponer a las madres durante la gestación a dietas ricas en carbohidratos o grasas saturadas se propicia la aparición del síndrome metabólico en las crías en su vida adulta y en muchos casos nacen con sobrepeso, por lo que empeora el panorama sobre su salud. Por ejemplo, se ha observado que las ratas alimentadas con dietas ricas en sacarosa tienden a desarrollar obesidad, esteatosis hepática y un aumento en los niveles de lipoproteínas de baja densidad (LDL); paradójicamente, estos animales presentaron una mayor sensibilidad a la insulina a nivel muscular con una tolerancia a la glucosa normal (Sedova et al., 2007). También dietas ricas en fructosa en las madres durante la gestación inducen en las crías durante la vida adulta la aparición de obesidad, hiperglucemia, hiperinsulinemia e hiperleptinemia (Vickers et al., 2011). Por otro lado, dietas con contenido alto en grasa saturada durante la gestación inducen una reducción del peso al nacer. Aunado a lo anterior, estos animales a largo plazo desarrollan obesidad, hipercolesterolemia, resistencia a la insulina e hipertensión. En modelos experimentales de primates no humanos se ha sugerido que un mayor estrés oxidativo podría estar relacionado con los efectos deletéreos de las dietas ricas en grasas saturadas durante la gestación (Maliqueo y Echiburú, 2014).

\section{Conclusiones}

En el último siglo se ha observado un aumento de las enfermedades crónicas no transmisibles en todo el mundo, y en donde las patologías asociadas al síndrome metabólico representan un importante problema de salud en México y el mundo. Entre los principales factores de riesgo que contribuyen a la pandemia del síndrome metabólico se reconocen a los malos hábitos alimenticios, el estilo de vida sedentario y los factores genéticos. Sin embargo, la dimensión del problema ha sugerido que existe otro factor sumamente importante en el origen de la pandemia del síndrome metabólico.

En la actualidad, se ha descrito a este factor imprescindible como la programación metabólica fetal y ha permitido plantear la hipótesis sobre los orígenes del desarrollo de la salud y las enfermedades en el adulto. De acuerdo con esta hipótesis, las alteraciones durante la vida perinatal condicionan cambios metabólicos adaptativos que producen modificaciones epigenéticas y propician la aparición de enfermedades asociadas al síndrome metabólico en la vida adulta cuando las condiciones ambientales no son adecuadas (exceso de calorías y falta de de ejercicio). Además, es muy importante resaltar que estas modificaciones en el ADN (epigenética) pueden ser heredadas a la descendencia.

\section{Análisis Prospectivo}

La mala nutrición materna relacionada con una ingesta inadecuada de nutrientes (desnutrición o sobrealimentación) durante los periodos críticos del desarrollo como el embarazo, la lactancia y los primero años de vida son un problema de salud en varios países del mundo, tanto desarrollados como en vías de desarrollo. En México y varios países del tercer mundo se ha observado que coexisten el desmedro (retraso en el crecimiento) y obesidad en las mismas poblaciones y en algunos casos en los mismos individuos (ENSANUT, 2016; Plaza Galarza, 2012). La coexistencia de estos dos fenómenos (desmedro y obesidad) en los mismos individuos y 
poblaciones tiene consecuencias alarmantes en la salud de la población adulta, por lo que se convierte en un factor importante en la pandemia del síndrome metabólico (Plaza Galarza, 2012).

Actualmente, México ocupa el primer lugar mundial en obesidad infantil y el segundo en obesidad en adultos (ENSANUT, 2016; Unicef, 2017). Este problema de salud sugiere que es necesario controlar la dieta de las madres de forma adecuada antes del embarazo, durante y en la lactancia para evitar que se produzcan cambios epigenéticos que puedan incrementar el riesgo de sufrir enfermedades metabólicas en la descendencia. No obstante, al ser una época de difícil intervención, es necesario realizar más estudios en animales, mejorar los estudios observacionales en personas e implementar políticas de salud pública al respecto. Sin embargo, aunque en estos tiempos la obesidad es un problema en México tanto en niños como en adultos, las políticas de salud están enfocadas sobre todo a tratar de prevenir el problema cuando los cambios epigenéticos muy probablemente ya están establecidos debido a la programación metabólica. Es muy importante tener en cuenta que la supervisión de una adecuada nutrición debe ser desde antes de la concepción, durante el embarazo y en los primeros años de vida de los hijos, así como realizar ejercicio regularmente, ya que, debido a las condiciones actuales en cuanto al tipo de alimentación muy rica en carbohidratos y grasas saturadas y a la falta de tiempo para actividades físicas, es muy fácil tener un ambiente favorable para que aparezcan enfermedades del síndrome metabólico. Las perspectivas sobre el panorama en cuanto a una mejora o reducción en las enfermedades crónicas no transmisibles tanto en México como a nivel mundial son poco alentadoras. Por lo tanto, no se debe esperar que un país establezca una adecuada política de salud pública, por lo que es muy importante comenzar con una concientización desde las familias sobre tener una buena alimentación basada en una adecuada cantidad de frutas y verduras y dejar a un lado los alimentos ricos en carbohidratos y grasas saturadas que, por desgracia, son los más apetecibles para la mayoría de la población.

\section{Agradecimientos}

Se agradecen los comentarios de los árbitros de la revista que mejoraron sustancialmente el contenido del artículo.

\section{REFERENCIAS}

Barba-Evia, J. R. (2018). México y el reto de las enfermedades crónicas no transmisibles. El laboratorio también juega un papel importante. Revista latinoamericana de patología clínica y medicina de laboratorio, 65, 4-17.

Barker, D. J., \& Osmond, C. (1986). Infant mortality, childhood nutrition, and ischaemic heart disease in England and Wales. The Lancet, 1, 1077-1081.

Barker, D. J. (1995). Fetal and infant origins of disease. European Journal of Clinical Investigation, 25, 457-63.

Barrera-Reyesa, R. y Fernández-Carrocera, L. A. (2015). Programación metabólica fetal. Perinatología y reproducción humana, 29(3), 99-105.

Burton, M. A., \& Lillycrop, K. A. (2019). Nutritional modulation of the epigenome and its implication for future health. Proceedings of the Nutrition Society, 19, 1-8.

Carpinello, O. J., DeCherney, A. H., \& Hill, M. J. (2018). Developmental origins of health and disease: The history of the barker hypothesis and assisted reproductive technology. Seminars in Reproductive Medicine, 36(3-04), 177-182.

ENSANUT (Encuesta Nacional de Salud y Nutrición). (2016). Informe final de resultados. Disponible en https:// www.gob.mx/cms/uploads/attachment/file/209093/ENSANUT.pdf.

Fleming, T. P., Watkins, A. J., Velazquez, M. A., Mathers, J. C., Prentice, A. M., Stephenson, J., Barker, M., 
Saffery, R., Yajnik, C. S., Eckert, J. J., Hanson, M. A., Forrester, T., Gluckman, P. D., \& Godfrey, K. M. (2018). Origins of lifetime health around the time of conception: Causes and consequences. The Lancet, 391(10132), 1842-1852.

Forsdahl, A. (1977). Are poor living conditions in childhood and adolescence an important risk factor for arteriosclerotic heart disease? British Journal of Preventive and Social Medicine, 31(2), 91-95.

Fowden, A. L., Giussani, D. A., \& Forhead, A. J. (2006). Intrauterine programming of physiological systems: Causes and consequences. Physiology (Bethesda), 21, 29-37.

GBD. Risk Factors Collaborators. (2017). Global, regional, and national comparative risk assessment of 84 behavioural, environmental and occupational, and metabolic risks or clusters of risks, 1990-2016: A systematic analysis for the Global Burden of Disease Study 2016. The Lancet, 390, 1345-1422.

Halfon, N., Forrest, C. B., Lerner, R. M., Faustman, E. M., Tullis, E., \& Son, J. (2018). Introduction to the Handbook of Life Course Health Developmenten, en N. Halfon, C. B. Forrest, R. M. Lerner, \& E. M. Faustman (eds.), Source Handbook of Life Course Health Development (pp. 1-16). Springer.

Hsu, C. N. y Tain, Y. L. (2019). The good, the bad, and the ugly of pregnancy nutrients and developmental programming of adult disease. Nutrients, 11(4). E894.

McCracken, E., Monaghan, M., \& Sreenivasan, S. (2018). Pathophysiology of the metabolic syndrome. Clinics in Dermatology, 36(1), 14-20.

Maliqueo, M. y Echiburú, B. (2014). Programación fetal de las enfermedades metabólicas. Revista de Farmacología de Chile, 7(1), 33-46.

Marciniak, A., Patro-Małysza, J., Kimber-Trojna, R. Z., Marciniak, B., Oleszczuk, J., \& Leszczyńska-Gorzelak, B. (2017). Fetal programming of the metabolic syndrome. Taiwanese Journal of Obstetrics and Gynecology, 2, 133-138.

Márquez-González, H., García-Sámano, V. M., Caltenco-Serrano, M., García-Villegas, E. A., Márquez-Flores, H. y Villa-Romer, A. R. (2012). Clasificación y evaluación de la desnutrición en el paciente pediátrico. El Residente, 7(2), 59-69.

Milagro, F. y Martínez, J. A. (2013). Epigenética en obesidad y diabetes tipo 2: papel de la nutrición, limitaciones y futuras aplicaciones. Revista Chilena de Endocrinología y Diabetes, 6(3), 108-114.

Patel, M. S., \& Srinivasan, M. (2011). Metabolic programming in the immediate postnatal life. Annals of Nutrition and Metabolism, 58(suppl 1), 18-28.

Perrone, S., Santacroce, A., Picardi, A., \& Buonocore, G. (2016). Fetal programming and early identification of newborns at high risk of free radical-mediated diseases. World Journal of Clinical Pediatrics, 5(2), 172-81.

Plaza Galarza, L. C. (2012). Desmedro, sobrepeso/obesidad y composición corporal en escolares de la ciudad de Riobamba (Tesis de posgrado). Ecuador: Escuela Superior Politécnica de Chimborazo.

Roseboom, T. (2019). Epidemiological evidence for the developmental origins of health and disease: Effects of prenatal undernutrition in humans. The Journal of Endocrinology, 242(1), T135-T144.

Sedova, L., Seda, O., Kazdova, L., Chylikova, B., Hamet, P., Tremblay, J., Kren, V., \& Krenova, D. (2007). Sucrose feeding during pregnancy and lactation elicits distinct metabolic response in offspring of an inbred genetic model of metabolic syndrome. American Journal of Physiology, 292, E1318-1324.

Skinner, M. (2008). What is an epigenetic transgenerational phenotype? F3 or F2. Reproductive Toxicology, 25, 2-6.

Tain, Y. L., Hsu, C. N. y Chan, J. Y. (2015). PPARs link early life nutritional insults to later programmed hypertension and metabolic syndrome. International Journal of Molecular Sciences, 17(1), E20. 
Taylor-Baer, M., \& Herman, D. (2018). From epidemiology to epigenetics: Evidence for the importance of nutrition to optimal health development across the life course, in N. Halfon, C. B. Forrest, R. M. Lerner, E. M. Faustman (eds.), Source Handbook of Life Course Health Development (pp. 431-462). Springer.

Unicef(United Nations International Children's Emergency Fund).2017. Centro deprensa: obesidad y sobrepeso. Fondo de las Naciones Unidas para la Infancia. Disponible en http://www.who.int/mediacen-tre/ factsheets/fs311/es/.

Velazquez, M. A., Fleming, T., \& Watkins, A.J. (2019). Periconceptional environment and the developmental origins of disease. The Journal of Endocrinology.

Vickers, M. H., Clayton, Z.E., Yap, C., \& Sloboda, D. M. (2011). Maternal fructose intake during pregnancy and lactation alters placental growth and leads to sex-specific changes in fetal and neonatal endocrine function. Endocrinology, 152, 1378-1387.

Wang, G., Bartel, T. R., \& Wang, X. (2018). Preconception and prenatal factors and metabolic risk, en N. Halfon, C. B. Forrest, R. M. Lerner, E. M. Faustman (eds.), Source Handbook of Life Course Health Development (pp. 1-13). Springer.

WHO (World Health Organization). (2015). Chapter 1: Burden: mortality, morbidity, and risk factors: World Health Organization. Retrieved from https://www.who.int/nmh/publications/ncd_report_full_en.pdf.

Zalts, H., \& Shomron, N. (2011). The impact of microRNAs on endocrinology. Pediatric Endocrinology Reviews, $8,354-362$.

CC BY-NC-ND 